\title{
The Origins of Two-State Spontaneous Membrane Potential Fluctuations of Neostriatal Spiny Neurons
}

\author{
Charles J. Wilson ${ }^{1}$ and Yasuo Kawaguchi ${ }^{2}$ \\ ${ }^{1}$ Department of Anatomy and Neurobiology, University of Tennessee, Memphis, Tennessee 38163, and 2Bio-Mimetic \\ Control Research Center, The Institute of Physical and Chemical Research (RIKEN), Rokuban, Atsuta-ku, \\ Nagoya 456, Japan
}

In vivo intracellular recordings of spontaneous activity of neostriatal spiny cells revealed two-state behavior, i.e., characteristic shifts of membrane potential between two preferred levels. The more polarized level, called the Down state, varied among neurons from -61 to $-94 \mathrm{mV}$. The more depolarized level, called the Up state, varied among neurons from -71 to -40 $\mathrm{mV}$. For any one neuron, the membrane potential in the Up and Down states was constant over the period of observation (from $15 \mathrm{~min}$ to $4 \mathrm{hr}$ ), and the cells spent little time in transition between states. The level of membrane potential noise was higher in the Up state than in the Down state. Spontaneous membrane potential fluctuations were not abolished by experimental alteration of the membrane potential, but the time spent in each state was altered when intracellular current was used to vary the baseline membrane potential.

Neither the sodium nor the calcium action potential that could be evoked by depolarization of spiny neurons was required for the occurrence of spontaneous shifts of membrane potential. Blockade of these action potentials using intracellular injection of QX314 and D890, respectively, altered neither the incidence of the membrane potential shifts nor the preferred membrane potential in either state. In contrast, antagonism of voltage-dependent potassium channels with intracellular cesium altered membrane potential shifts. In the presence of QX314 and D890, intracellular injection of cesium caused little or no change in the Down state and a large depolarizing shift in the Up state (to about $-20 \mathrm{mV}$ ). Under these circumstances, the neuron responded to current in a nearly linear manner, and membrane conductance was found to be increased in the Up state, attributable to a membrane conductance with the same reversal potential as that of the synaptic potential evoked by cortical stimulation.

These results indicate that the event underlying the Up state is a maintained barrage of synaptic excitation, but that the membrane potential achieved during the Up state in neostriatal spiny neurons is determined by dendritic potassium channels that clamp the membrane potential at a level determined by their voltage sensitivity. Neostriatal spiny neurons ordinarily receive enormously powerful excitation, which would drive the cells to saturation, and probably destroy them, if it were not for these potassium currents.

Key words: inward rectification; QX314; D890; intracellular recording; basal ganglia; synaptic integration
When recorded in vivo, neostriatal spiny neurons show a characteristic pattern of spontaneous activity consisting of long periods of silence separated by brief episodes of firing (for review, see Wilson, 1993). During performance of learned motor behaviors, similar episodes of firing and silent periods become entrained to particular phases of the movement (Hikosaka et al., 1989; Kimura et al., 1990; Aldridge and Gilman, 1991), indicating that this pattern of firing is a fundamental feature of information processing in the neostriatum, and not an artifact of experimental details such as anesthesia.

Intracellular recordings of neostriatal spiny neurons in immobilized, locally anesthetized rats (Wilson and Groves, 1981) and in urethane-anesthetized rats (Wilson, 1993) have shown that the episodic firing pattern is attributable to the occurrence of $0.1-3$ sec periods of sustained depolarization in the spiny neurons, which require the integrity of excitatory inputs from the cerebral cortex and thalamus. Thus, in slices of the neostriatum maintained

\footnotetext{
Received March 6, 1995; revised Aug. 4, 1995; accepted Aug. 4, 1995.

This work was supported by Office of Naval Research Grant N00014-92-J-1113 and Public Health Service Grant NS20743 (C.J.W.), and by the Frontier Research program (Y.K.).

Correspondence should be addressed to Charles J. Wilson, Department of Anatomy and Neurobiology, University of Tennessee, Memphis, TN 38163.

Copyright $(1996$ Society for Neuroscience $0270-6474 / 96 / 162397-14 \$ 05.00 / 0$
}

in vitro, or in animals with large cortical and thalamic lesion, the membrane potentials of the cells correspond to that of the hyperpolarized periods seen in vivo, and no depolarizing episodes are seen (Wilson et al., 1983; Calabresi et al., 1990; Wilson, 1993). On this basis, it was concluded that the hyperpolarized periods were not attributable to the action of tonic inhibitory circuits but, rather, were caused by the lack of sufficient excitation. The depolarizing episodes were attributed to maintained, coordinated synaptic excitation from the cerebral cortex and thalamus (Wilson and Groves, 1980; Wilson et al., 1983; Calabresi et al., 1990).

An explanation of the membrane potential shifts of spiny neurons based solely on patterned synaptic excitation must struggle to explain some of the characteristic features of the membrame potential changes. Most obvious of these is the constancy of the membrane potential in both the hyperpolarized and the depolarized episodes. Unless excitatory input to the neostriatal neurons was very tightly constrained, so that it could maintain a constant rate over a period of seconds, it is difficult to understand why the depolarized state would remain constant within a few millivolts over such a long period. Given that neostriatal projection neurons receive several thousand excitatory inputs from nearly as many different afferent neurons (Wilson, 1994), this would imply a remarkable coordination of inputs. Additionally, the abrupt na- 
ture of onset and offset of the depolarizations must be explained by very rapid recruitment of a large number of excitatory inputs that are to participate in the depolarizing episode. These features of the membrane potential shifts suggest the existence of two discrete membrane potential states, rather than simply a reflection of the constantly changing complement of synaptic input to the spiny neuron.

In an attempt to explain this two-state behavior of the neostriatal projection neuron, Wilson (1992) suggested that the constancy of the hyperpolarized state of the membrane (which will be called the Down state) was attributable to the activation of a strong and rapidly activating inwardly rectifying potassium-selective current known to be present in these cells (Kita et al., 1984; Kawaguchi et al., 1989; Uchimura et al., 1989; Jiang and North, 1991; Nisenbaum and Wilson, 1995). When the volume of excitatory input falls below the amount required to maintain the depolarized state (and to deactivate the inwardly rectifying $\mathrm{K}^{+}$current), it was proposed that the cell would be clamped near the $\mathrm{K}^{+}$equilibrium potential by the potassium-selective inward rectifier. The depolarized state (which will be called the Up state) was attributed to the interaction between excitatory input and voltage-dependent $\mathrm{K}^{+}$currents activated at membrane potentials near, but slightly below, spike threshold. The presence of such currents in neostriatal spiny neurons is well documented (Kita et al., 1985c; Bargas et al., 1989; Calabresi et al., 1990b; Surmeier et al., 1991, 1992; Nisenbaum and Wilson, 1995; Galarraga et al., 1994; Nisenbaum et al., 1994).

Experimental testing of this hypothetical mechanism for the subthreshold two-state behavior of the neostriatal spiny neuron is not accessible in vitro, because there is currently no in vitro preparation in which this activity is maintained. Although the activation and deactivation of currents postulated to be responsible for the membrane potential states can be and have been studied in dissociated neostriatal cells and in neostriatal slices (Surmeier et al., 1992; Nisenbaum et al., 1994; Nisenbaum and Wilson, 1995), it remains to be shown that these currents, and not some other mechanism, are responsible for these phenomena in vivo. In the experiments described here, spontaneous shifts in the membrane potentials of spiny neurons were studied in vivo, using intracellular application of cesium to block potassium currents after blockade of sodium and calcium currents using QX314 and D890, respectively. These experiments provide a direct test of the hypothesis that voltage-sensitive $\mathrm{K}^{+}$conductances are responsible for the existence of discrete subthreshold membrane potential states in neostriatal spiny neurons.

\section{MATERIALS AND METHODS}

Intracellular recordings were performed in Long-Evans rats anesthetized with urethane $(1.5 \mathrm{mg} / \mathrm{kg})$ supplemented by hourly intramuscular injections of a combination of ketamine and xylazine $(35 \mathrm{mg} / \mathrm{kg}$ ketamine, 7 $\mathrm{mg} / \mathrm{kg}$ xylazine). The animals were placed in a stercotaxic device and suspended by a tail clamp to reduce breathing movements, but were not artificially respired. Temperature was maintained at $37 \pm 0.5^{\circ} \mathrm{C}$ using a feedback-controlled heating pad. Stimulating electrodes, consisting of pairs of stainless steel insect pins ( 000 gauge) insulated except for the tips, were placed $0.5-0.75 \mathrm{~mm}$ apart in the cerebral peduncle at the level of the pons, in the ipsilateral substantia nigra, in the contralateral cerebral cortex, or in the contralateral neostriatum. These were used for antidromic identification of striatonigral neurons or for orthodromic synaptic activation of the cells. The cortex overlying the neostriatum from a level $8.5-12.5 \mathrm{~mm}$ anterior to the interaural line and $0.5-3.5 \mathrm{~mm}$ lateral to the midline was exposed. Recording microelectrodes were pulled from 3.0 $\mathrm{mm}$ smooth-bore glass stock. They had shank lengths of 5-10 $\mathrm{mm}$ and tips broken under microscopic control to have tips from $0.1-0.5 \mu \mathrm{m}$ in diameter (as judged using interference colors under epi-illumination).
The microelectrodes had resistances ranging from 30 to $100 \mathrm{M} \Omega$ when filled with $1 \mathrm{M} \mathrm{K}$-acetate and measured in the brain. Microelectrodes were filled with $4 \%$ biocytin in $1 \mathrm{M} \mathrm{K}$-acetate, 1 or $2 \mathrm{M} \mathrm{K}$-methylsulfate, $1 \mathrm{M}$ $\mathrm{KCl}, 2 \mathrm{M} \mathrm{Cs}$-acetate, or $2 \mathrm{M} \mathrm{CaCl}$. In addition, the electrolyte sometimes contained 10-50 mM QX314 or 2-10 mM D890 (Research Biochemicals, Natick, MA), or both, to block inward currents attributable to voltagesensitive sodium or calcium currents, respectively. Intracellular record ings were obtained using a conventional active bridge amplifier, digitized (usually at $4 \mathrm{kHz}$ ), and stored digitally for later analysis. Electrodes were tested for linearity during the experiment by passage of $\pm 1.5 \mathrm{nA}$ currents, and data collected using electrodes failing this linearity test were not included in the sample.

After completion of the experiment, neurons were stained with biocytin by passage of $0.5-2 \mathrm{nA}$ depolarizing current pulses, $350 \mathrm{msec}$ on $/ 350 \mathrm{msec}$ off, for 15-30 min. Animals were killed by an overdose of urethane and perfused intracardially with $4 \%$ formaldehyde in $0.15 \mathrm{M}$ phosphate buffer. Sections through the region of the recording were cut using a vibratome and stained using the avidin-biotin-horseradish peroxidase method as described by Horikawa and Armstrong (1988).

\section{RESULTS}

\section{Spontaneous membrane potential fluctuations with control electrolyte}

Thirty-eight neostriatal spiny neurons, including 11 neurons the axons of which were traced to the substantia nigra, and 5 of which were observed to terminate in the globus pallidus, were studied using electrodes filled with control solutions ( $1 \mathrm{~m} \mathrm{~K}$-acetate, $2 \mathrm{~m}$ $\mathrm{K}$-methylsulfate, or $1 \mathrm{M} \mathrm{KCl}$ ). An example showing the typical appearance of one of these cells after injection with biocytin is shown in Figure 1. The morphological features of the neurons matched those of spiny neurons as described in previous studies (Wilson and Groves, 1980). An example showing spontaneous activity of one of these cells is shown in Figure 2. In all control cells, the membrane potential switched between two relatively constant subthreshold levels.

Quantification of the membrane potential fluctuations was accomplished as illustrated in Figure 3. The histograms shown there, and others like them in subsequent figures, were constructed by sampling spontaneous activity at $4 \mathrm{kHz}$ and counting the number of samples at each membrane potential. This gives the proportion of the time spent by the neuron at each membrane potential. In the absence of injected current, the neurons spent most of their time at membrane potentials near two distinctly different values. One, the Down state potential, was about $-75 \mathrm{mV}$ in Figures 2 and 3 , and the other, the Up state, was about $-54 \mathrm{mV}$. Much less time was spent at intermediate membrane potentials, which reflects the rapid transitions made between the two states. The variation in membrane potential in the Down state was much less than that of the Up state, which corresponds to the difference in noise in the two states seen in individual traces. In addition, action potentials were intermittently triggered by the largest of the noisy membrane potential fluctuations in the Up state. The very small tail in the distribution to the right of the Up state seen in the third histogram in Figure $3(+0.75 \mathrm{nA})$ was attributable to action potentials. Even when action potentials were numerous, their short durations caused their contribution to the histograms to be minimal.

Stable recordings made over the course of $2-4 \mathrm{hr}$ in some control neurons showed very little change in the histngrams like those in Figure 3, or in the preferred membrane potential in the Up and Down states. Among cells, however, there was some variability in the preferred membrane potentials. The Down state varied from -61 to $-94 \mathrm{mV}$ among cells, and the Up state likewise varied from -40 to $-71 \mathrm{mV}$. Mean and SD values for $\mathrm{Up}$ and Down states are shown in Table 1. 


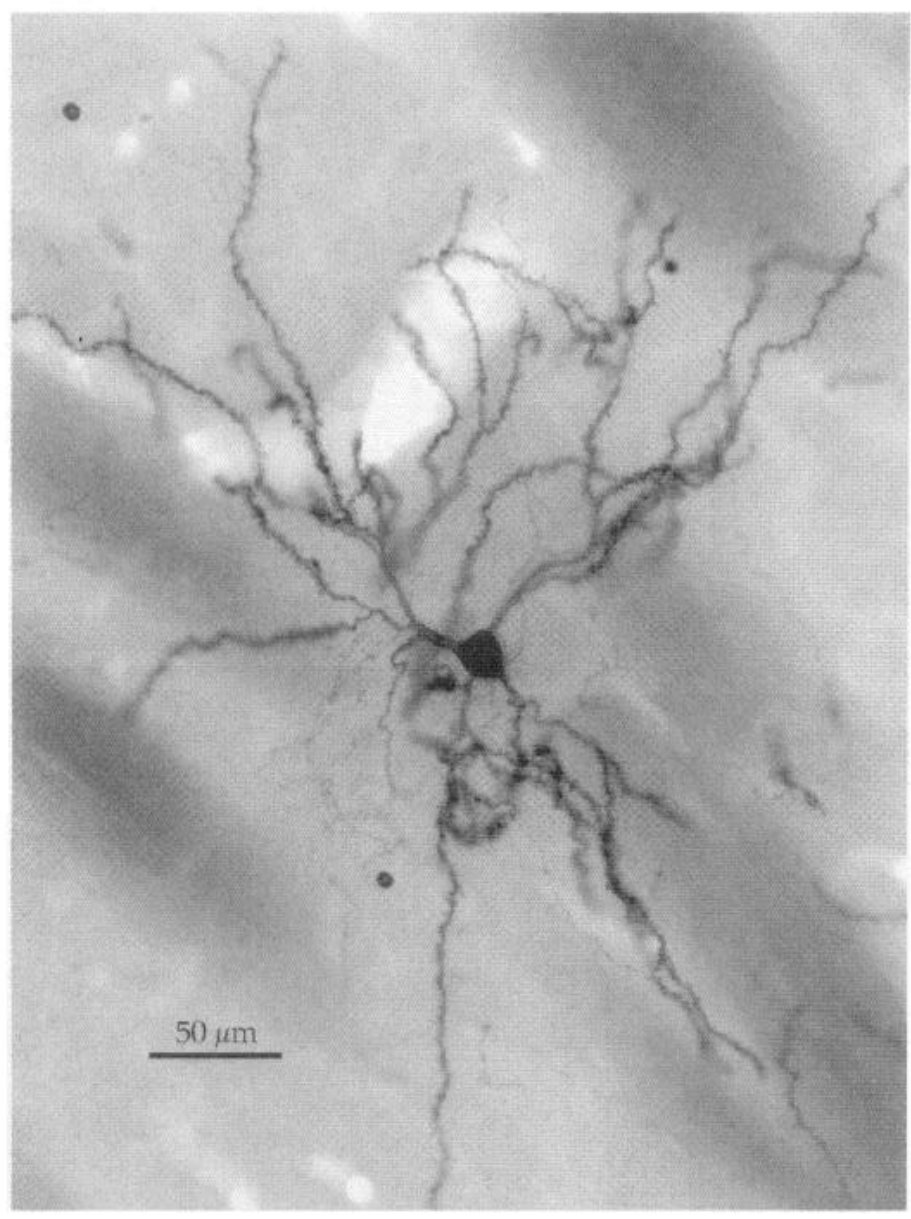

Figure 1. Photomontage of a spiny neuron recorded in vivo and stained intracellularly with biocytin. Dendritic spines are visible on the distal dendrites, whereas the soma and proximal dendrites were free of spines. Local axon collaterals are visible. The background staining is attributable to counterstaining with osmium tetroxide. Intracellular recording using electrodes filled with QX314, D890, and cesium had no visible effect on the morphological features of spiny neurons.

It is likely that some of the apparent variation in absolute membrane potential from cell to cell was an artifact of microelectrode tip potentials. Many of the recordings were maintained over a period of hours, during which time the cell was subjected to a variety of tests and current injections, including the large (1-2 nA) current injections made to inject biocytin into the cell. DC offset was nulled before impaling each neuron, and membrane potential measurements were corrected for tip potential using the extracellular potential recorded immediately after withdrawal from the cell, which sometimes was as much as $3 \mathrm{hr}$ after the spontaneous activity measurements were made. When, as occasionally occurred, there was no measurable tip potential just before impaling a neuron but one was detected after the recordings were completed, there was necessarily some uncertainty about the accuracy of DC measurements taken during the intervening time. It is possible to gain an estimate of the error in absolute membrane potential measurement arising from uncompensated tip potentials, by comparing the Down and Up state membrane potentials, peak spike amplitude, and spike thresholds. This comparison showed that the cell-to-cell variation in membrane potential observed in these experiments was not attributable entirely to uncompensated tip potentials. For example, the difference between the Up and Down states was no less variable than either alone,

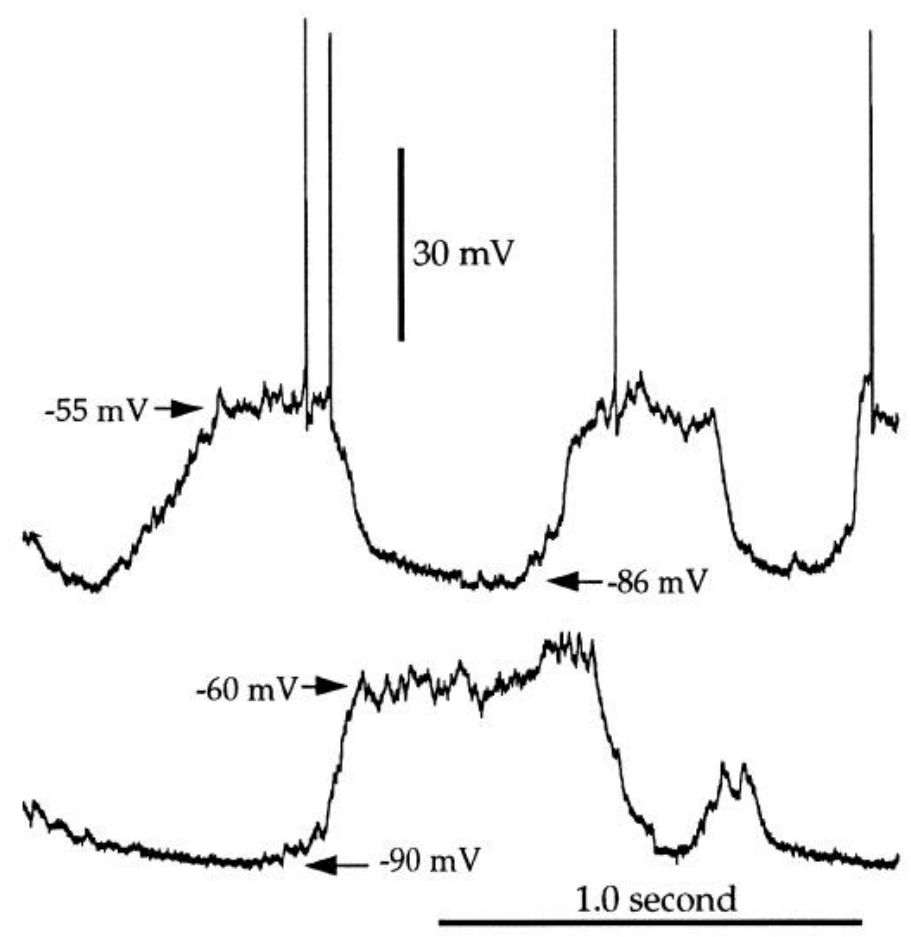

Figure 2. Membrane potential fluctuations in a neostriatal spiny cell recorded using electrodes filled with $1 \mathrm{M} \mathrm{K}$-acetate and identified by intracellular staining with biocytin. The membrane potential exhibited episodes of relatively constant hyperpolarization interrupted by depolarizing episodes. Membrane potential noise was greater during the depolarization, and action potentials were triggered occasionally by the noise. The depolarizing episodes were often, but not always, rhythmic in their occurrence. The top trace shows membrane potential in the absence of applied current. In this and subsequent slow traces, spike amplitude varies somewhat because of the $4 \mathrm{kHz}$ sampling frequency. In the bottom trace, a small, constant hyperpolarizing current $(0.1 \mathrm{nA})$ was passed through the electrode to prevent action potentials. The rectangular appearance of the transitions between membrane potentials is apparent despite the absence of action potentials.

varying from 9 to $30 \mathrm{mV}$ (mean $=22+6.9 \mathrm{mV}$ ), but there was a moderate positive correlation between Up state and Down state membrane potentials $(r=0.67, \mathrm{df}=35, p<0.01)$. Although consistent with the presence of some variance attributable to uncompensated tip potential, these results fail to account for more than about half of the cell-to-cell variation in Down and Up state membrane potentials in this way. A similar cell-to-cell variation in resting membrane potential is seen in striatal slices (Nisenbaum and Wilson, 1995), where it is most likely attributable to variation in intrinsic membrane properties of the cells, because synaptic input is absent in that preparation. An alternative possibility, that membrane potential is strongly under the control of anesthetic level, was ruled out by the absence of any change of membrane potentials after administration of anesthetic supplements.

Previous studies have shown that the occurrence of the Up state absolutely required the presence of excitatory synaptic input to the neostriatal neurons (Wilson, 1993). These studies did not rule out the participation of active membrane properties of neostriatal neurons either in the transition to the Up state, or in maintaining that membrane potential state once achieved. To test for a possible voltage dependence of the Up state, spontaneous membrane potential shifts were studied during the passage of constant current to depolarize or hyperpolarize the membrane of the neostriatal neuron. If the spontaneous membrane potential shifts 

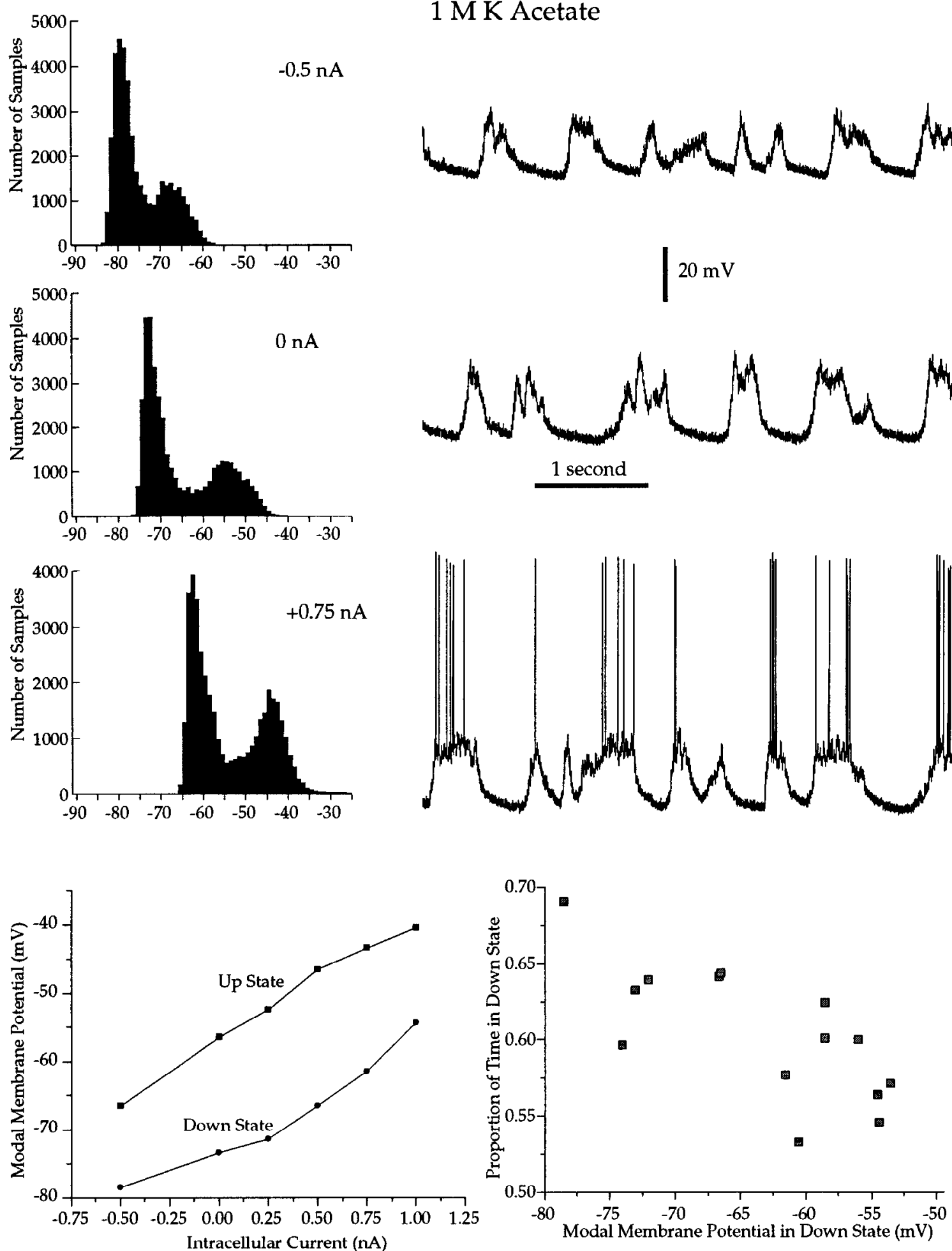

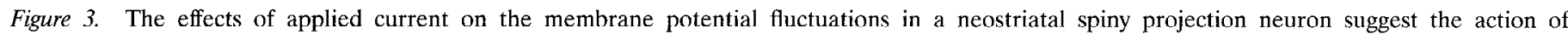

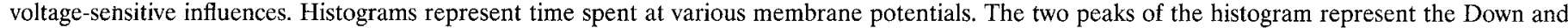

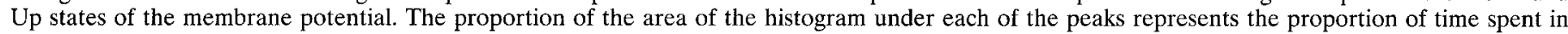

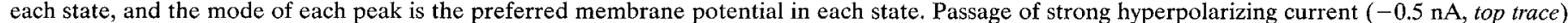

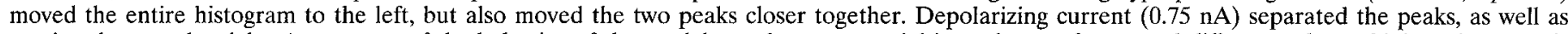

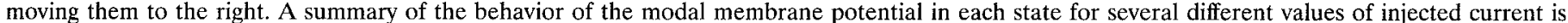

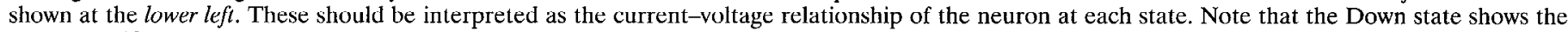

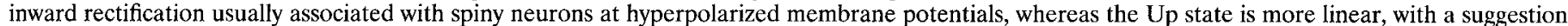

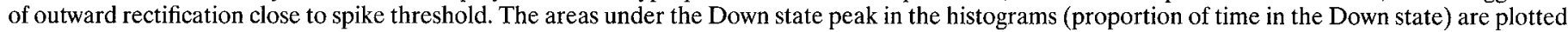

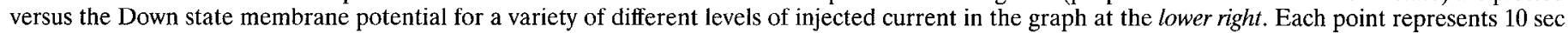
of membrane potential sampled at $4 \mathrm{kHz}$. 
Table 1. Properties of Up and Down states of spiny neurons

\begin{tabular}{|c|c|c|c|c|c|}
\hline & Control & QX314 & $\mathrm{QX} 314+\mathrm{D} 890$ & Cesium & $\begin{array}{l}\text { QX314 + D } 890 \\
+ \text { cesium }\end{array}$ \\
\hline$n$ & 38 & 14 & 15 & 5 & 6 \\
\hline$V_{\mathrm{M}} \mathrm{Up}(\mathrm{mV})$ & $-54.2 \pm 6.9$ & $-58.1 \pm 11.2$ & $-42.7 \pm 20.0$ & - & $-31.5 \pm 18.9$ \\
\hline$V_{\mathrm{M}} \operatorname{Down}(\mathrm{mV})$ & $-76.6 \pm 9.2$ & $-77.2 \pm 9.7$ & $-69.9 \pm 15.4$ & $-75.6 \pm 12.8$ & $-64.3 \pm 9.9$ \\
\hline$R_{\mathrm{N}}(\mathrm{M} \Omega)$ & $23.9 \pm 9.9$ & $31.3 \pm 10.3$ & $26.5 \pm 5.9$ & & $46.8 \pm 16.8$ \\
\hline
\end{tabular}

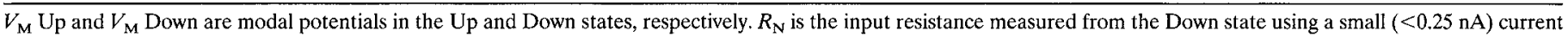
pulse.

-, Not measured.

simply were a reflection of the envelope of synaptic excitation impinging on the neostriatal neuron, intracellular current would shift the values of the Up and Down states in proportion to the input resistance of the neuron at those two states, but would not alter the time spent by the cell in the two states. This experiment was performed for 24 neurons recorded using electrodes filled with control electrolyte ( $1 \mathrm{M}$ K-acetate or 1 or $2 \mathrm{M}$ $\mathrm{K}$-methylsulfate). An example of the results of this experiment are shown in Figure 3. Hyperpolarizing and depolarizing current shifted the distribution of membrane potentials, but did not affect the Up and Down states identically. To analyze the differential effects on the membrane potential for each state, the modal value of membrane potential at each state was obtained from the peaks of histograms like those shown in Figure 3. The effect of constant current on the modal membrane potential in the Up and Down states is shown at the bottom left in Figure 3. The Down state showed the characteristic inward rectification described in neostriatal spiny neurons in slices (Kawaguchi et al., 1989; Nisenbaum and Wilson, 1995), whereas the Up state current-voltage $(I-V)$ curve was more nearly linear but showed some indication of outward rectification at potentials positive to about $-50 \mathrm{mV}$. To measure the proportion of the time spent in each state, the histograms were divided into $U p$ and Down portions at the minimum point between the peaks (or at the midpoint between peaks in a few cases in which there was a broad valley between the peaks), and the area under the curve for each state was calculated. This area represents the total time spent in each state. The result for the cell in Figure 3 was typical of cells recorded using electrodes filled with control electrolyte, and is shown at lower right in Figure 3. In that figure, the time spent in the Down state is plotted for each of several data samples taken at various levels of injected current. The effect of membrane rectification was removed from the plot by comparing the time in the Down state with the modal membrane potential in the Down state, rather than directly with injected current. In all cases, depolarization by injected current decreased the time spent in the Down state whereas hyperpolarization increased it. This result was not dependent on the use of the minimum point to divide the Up and Down states. If the samples were divided using midpoint between the peaks rather than by the minimum point, the results were the same. It was not possible to force the cell permanently into the Down state by hyperpolarizing it, but very strong hyperpolarizing currents could often transform the Up state to a period of noisy and irregular depolarization, with no preferred membrane potential. Strong constant depolarizing currents, at least up to the point at which cells fired continuously, likewise could not force the cells into a continuous Up state. The proportion of time spent in the Down state was adjustable from $\sim 70-50 \%$ over the range of currents that could be passed under the conditions of the experiment.
Although some rhythmicity was nearly always present in these transitions in anesthetized animals, there was usually a strong aperiodic component as well. This somewhat unpredictable pattern of transitions to the Up state is reflected in the sample-tosample variation in Up state time, seen as scatter among the points in the graph.

The temporal pattern of transitions to the Up state probably reflects the complex pattern of synaptic input to the striatal neurons, but the effect of constant current on time spent in the UP state suggests that the membrane potential shifts are partly determined by voltage-dependent mechanisms and not simply by the pattern of excitatory synaptic input. This conclusion was also suggested by the highly stereotyped trajectory of the membrane potential during the transition from the Down to the Up state and from the Up to the Down state. Examples of several such transitions superimposed are shown in Figure 4. The transition to the Up state was relatively rapid, highly reproducible, and sigmoidal in shape. The transitions to the Down state took longer to complete and exhibited a gradual settling of the potential in the Down state. One possible explanation for the differences seen in the transitions to the Up and Down states is that they reflect changes in the effective membrane time constant attributable to activation and deactivation of voltage-sensitive currents engaged by the changes in membrane potential.

As an initial attempt to detect the influence of voltagedependent conductances in the membrane potential state transitions, the responses of spiny neurons to current pulses in the Up and Down states were compared. Examples showing the typical

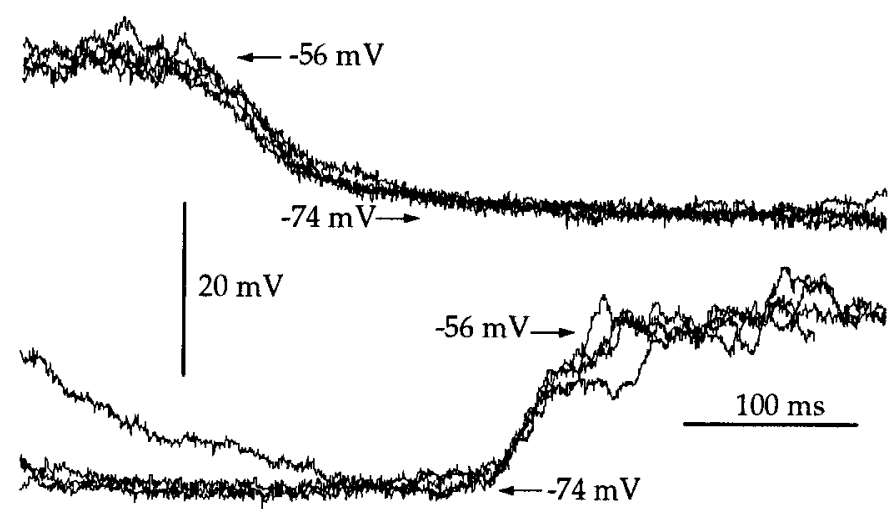

Figure 4. Superimposed traces showing that the transition to Up and Down states has repeatable and stereotypical shapes. All traces are from a single 5 sec sample from the same neuron. Five transitions are shown superimposed in each case. Transitions to the Up state were more abrupt than transitions to the Down state, which have a more exponential decay to the more polarized membrane potential. Transitions shown are from the middle of traces in Figure 3. 


\section{M KAcetate}

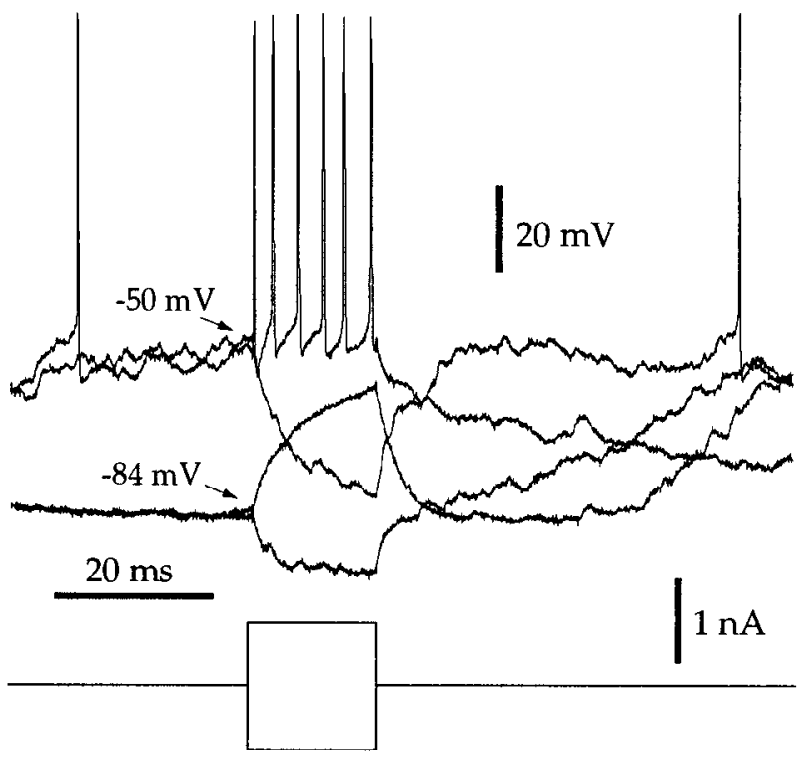

$25 \mathrm{mM}$ QX314, $1 \mathrm{mM}$ D890 in $2 \mathrm{M}$ KAcetate

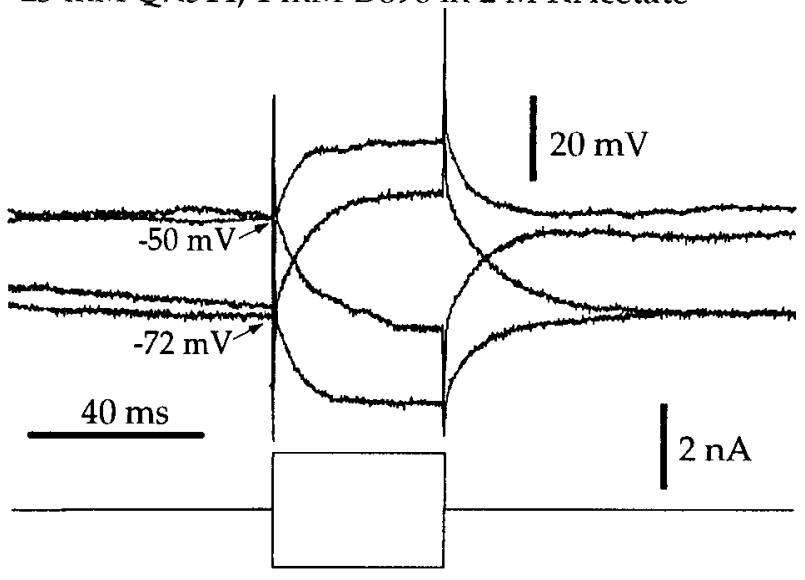

Figure 5. The whole neuron input resistance of the spiny neuron differs in the Up and Down states and depends on the polarity of current injection. The top traces are from a control recording, and the bottom traces are from a cell in which spikes were blocked using QX314 and D890. Two traces from the Up state and two traces from the Down state are shown in each. In each state, one trace shows the response to a hyperpolarizing and one to a depolarizing current pulse. Traces were selected for their constant membrane potential before and during the current pulse. Spontaneous membrane potential fluctuations after the pulse was over did not depend on the polarity or strength of the current pulsc. In the Down state, the input resistance was higher and the time constant was longer in response to depolarizing current, indicating the influence of inwardly rectifying currents in the Down state. In the Up state, input resistance was high and the time constant was long in response to hyperpolarizing current. Spikes obscure the response to depolarizing current in the Up state in the control cell, but with blockade of spikes, a low input resistance and short time constant are apparent.

response of neostriatal spiny neurons to current pulses in vivo are shown in the top traces of Figure 5 for two sweeps taken from the Up state and two taken from the Down state. In the Down state, a strong inward rectification was apparent from comparison of the response to depolarizing and hyperpolarizing current pulses. In the Up state, depolarizing pulses often triggered action potentials, so that no estimate of input resistance could be obtained in that way. The response to hyperpolarizing pulses from the Up state approximated that to depolarizing pulses in the Down state. For the group of 21 control neurons tested in this way, input resistance measured with hyperpolarizing pulses was less in the Down state $($ mean $=23.9 \pm 9.9 \mathrm{M} \Omega$ ) than in the Up state $($ mean $=32.7 \pm$ $13.9 \mathrm{M} \Omega ; t=4.7, \mathrm{df}=21, p<0.01$ ). It was not possible to test with depolarizing pulses, because of interference with action potentials in the Up state, as shown in Figure 5. It is probable that the difference between Up and Down states would have been reversed if the input resistance were measured using depolarizing pulses, because of outward rectification. When action potentials were blocked using QX314 and D890, application of depolarizing pulses clearly revealed outward rectification in the Up state (Figure 5, bottom traces).

\section{Blockade of action potentials with QX314 and D890}

The lack of an effect of hyperpolarization on the occurrence of membrane potential transitions ruled out a direct effect of action potential currents on the transitions themselves. However, to examine the possible role of other depolarization-activated currents in limiting the voltage in the Up state, blockade of action potentials was required. QX314 $(10,25$, or $50 \mathrm{~mm})$ was added to the electrolyte (1 M K-acetate or $1 \mathrm{~m}$ K-methylsulfate) to prevent action potentials in the recorded neurons. The blockade of action potentials by QX314 was similar to that reported for other central neurons (Connors and Prince, 1982; Butterworth and Strichartz, 1990). At the lowest concentration used $(10 \mathrm{~mm})$, complete block of fast action potentials did not occur, but action potentials were reduced in amplitude and repetitive firing in response to constant current pulses failed after one or two spikes. Under these conditions, slow depolarizing potentials often followed the partial fast spikes, which triggered slow afterhyperpolarizations not normally seen in neostriatal spiny neurons. Examples showing the effect of partial and total fast spike blockade with OX314 are shown in Figure 6. After complete blockade of fast action potentials with higher doses or longer exposure to QX314, the slow depolarizing potentials were not seen unless the membrane potential was depolarized further by large current injections through the microelectrode (Fig. 6). Because the slow depolarizing potentials resembled the high-voltage-activated calcium spikes reported in neostriatal spiny neurons recorded in slices (Kita et al., 1985), an attempt was made to prevent their occurrence by injection of D890, a quaternary derivative of methoxyverapamil (Deisz and Prince, 1987; Valdivia and Coronado, 1990). Including 1, 2, or 10 mM D890 in the microelectrode abolished spontaneous, slowdepolarizing potentials and raised the threshold for slow potentials evoked by current pulses. These effects were achieved very rapidly after impaling spiny neurons, but a complete blockade of slow depolarizing potentials evoked by current pulses was attained only after prolonged recording with D890-containing electrodes ( $1 \mathrm{hr}$ or more). Increasing the concentration of D890 beyond 2 mM accelerated the rate at which the block occurred, but did not produce a more complete block of slow depolarizing potentials. At the two highest concentrations ( 2 and $10 \mathrm{~mm}$ ), D890 also blocked inward rectification in some neostriatal spiny neurons. Thus, the lowest D890 concentrations were preferred. Combined use of QX314 and D890 was required to study the contribution of cesium-sensitive potassium currents to the state transitions. Cesium-containing electrodes had profound effects, increasing action potential duration in the absence of blockers of inward currents (Fig. 6). Cesium greatly increased the frequency of occurrence and extended the durations of the slow depolarizing potentials and interfered with measurement of spontaneous mem- 

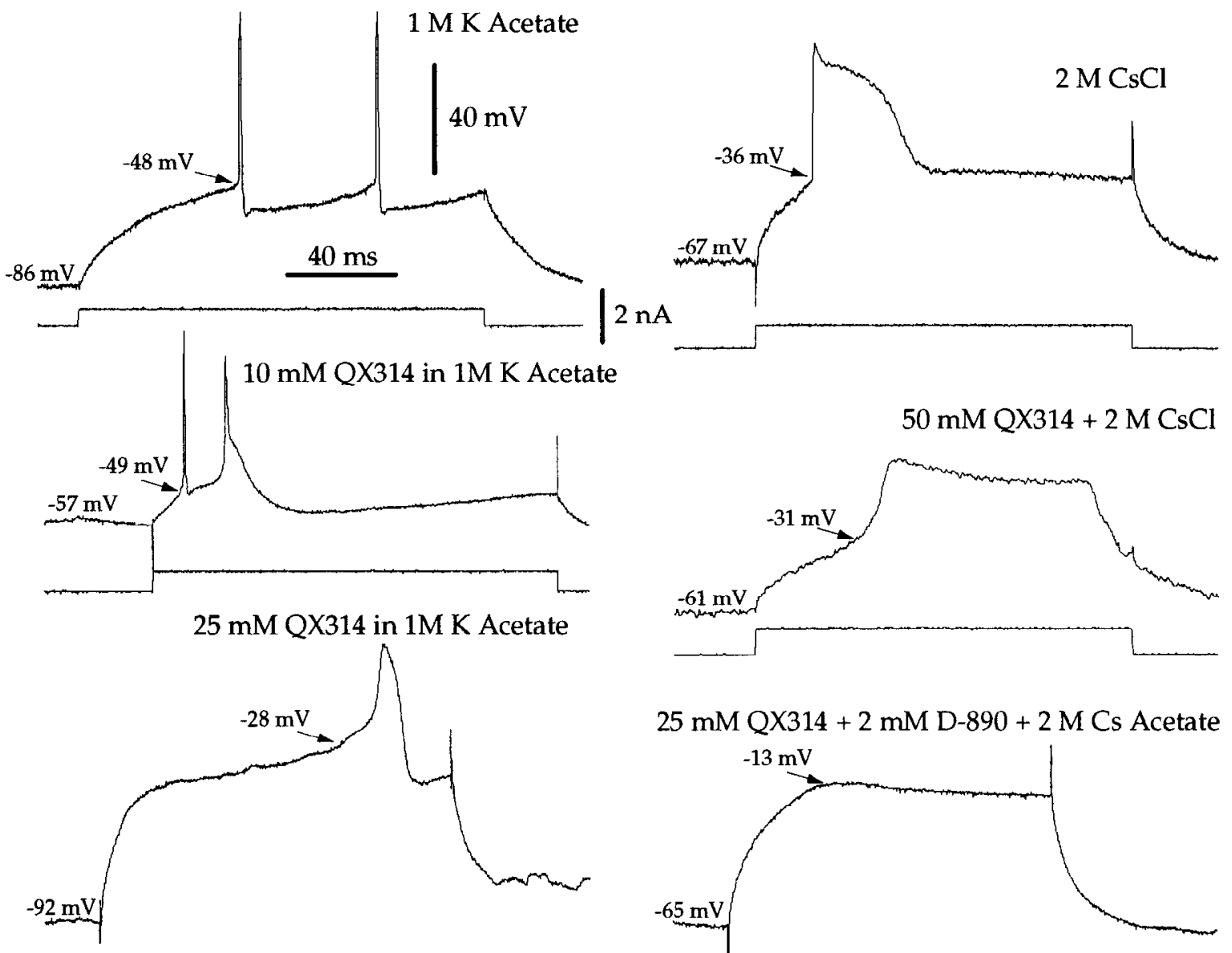

$25 \mathrm{mM}$ QX314 + $2 \mathrm{mM}$ D-890 + $2 \mathrm{M}$ Cs Acetate
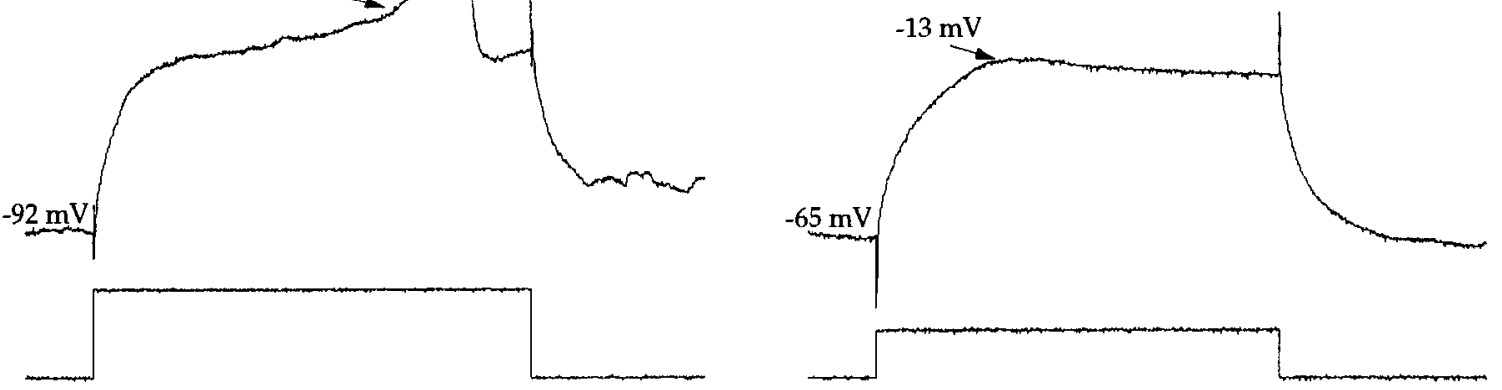

$25 \mathrm{mM} Q \times 314+2 \mathrm{mM}$ D890 $+2 \mathrm{M}$ Cs Acetate
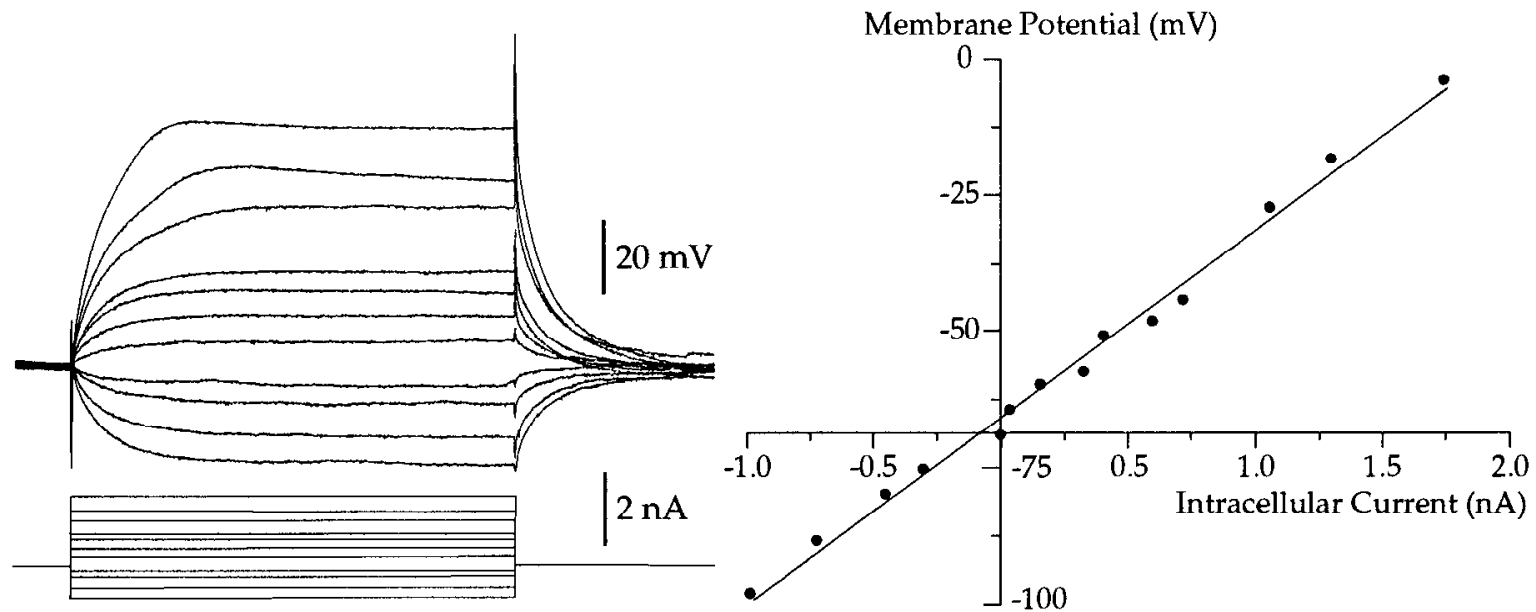

$40 \mathrm{~ms}$

Figure 6. Effects of intracellular cesium, QX314, and D890 on responses to depolarizing current pulses in a neostriatal neuron in vivo. The response to current applied in the Down state using control electrolyte $(1 \mathrm{~m} \mathrm{~K}$ acetate $)$ was similar to that normally observed in neostriatal tissue slices. Addition of a low concentration of $Q X 314(10 \mathrm{~mm})$ to the electrolyte reduced action potential amplitude and allowed the occurrence of wide, low-amplitude spike afterpotentials. Spikes triggering these depolarizing afterpotentials also exhibited subsequent spike afterhyperpolarizations (middle left). A higher dose of QX314 (25 mM) blocked fast action potentials. At much higher levels of depolarization, slow QX314-insensitive action potentials could be evoked, and

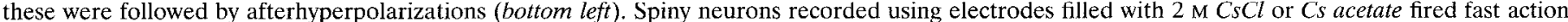
potentials with very long durations, but without afterhyperpolarizations (top right). Addition of QX314 to this electrolyte greatly slowed the fast onset of these action potentials and raised their threshold (middle right). No spike afterhyperpolarizations were observed (not shown). Addition of D890 to the electrolyte produced nearly linear membrane responses in spiny neurons (bottom right). A small, slowly activating outward rectification was observed at highly depolarized levels. A more complete set of traces in this condition is shown below, along with a plot of the steady-state current-voltage curve. 
brane potential shifts (Fig. 6). Cesium injection also abolished the long-lasting hyperpolarization that normally followed the slowdepolarizing potentials (Fig. 6). Injection of QX314 at concentrations that completely blocked fast action potentials slowed, but did not prevent, the long-duration depolarizing responses seen in cells recorded with cesium-containing electrodes. Combined blockade of inward currents using QX314 and D890 was required to prevent interference of regenerative depolarizing potentials in the measurement of spontaneous membrane potential shifts. A combination of QX314, D890, and cesium led to a greatly linearized neostriatal spiny neuron, although some residual outward rectification was apparent at the most depolarized potentials, as shown at the bottom in Figure 6. The responses of spiny neurons recorded in this way closely resembled those reported for the same cells in slices poisoned using tetrodotoxin, cadmium, and high doses of tetraethylammonium (Nisenbaum and Wilson, 1995).

It should be noted that QX314, D890, and cesium are not highly selective treatments. The blockade of inward rectification by $\mathrm{D} 890$ sometimes observed in spiny neurons is especially illustrative and should be added to concerns about possible effects of QX314 on potassium currents (Andreasen and I Iablitz, 1993) and on the wide spectrum of channels that are affected by intracellular cesium. The interpretation of their effects on spontaneous membrane potential fluctuations was most straightforward when the effects were negative. The effectiveness of QX314 and D890 in blocking regenerative currents (shown in Fig. 6) and the effects of cesium on both inward and outward rectification were tested using current pulses applied in the Down state at various times during the recording of spontaneous membrane potential fluctuations. These provided positive controls showing that the treatments were blocking (at least) these currents at the time their effects on spontaneous activity were assessed. The composition of ionic currents making up the action potential and its afterpotentials in spiny neurons has been studied in detail using spiny neurons in slices (Calabresi et al., 1990b; Pineda et al., 1992; Kita et al., 1995b), and the purposes of Figure 6 are (1) to show that the present results are consistent with the known properties of these neurons, and (2) to show that the effects of cesium, QX314, and D890 are as expected from those properties.

\section{Spontaneous membrane potential shifts in the absence of action potentials}

Blockade of action potentials, whether with QX314 alone or with a combination of QX314 and D890, had no measurable effect on the occurrence of membrane potential shifts or on the preferred membrane potential in the Up or Down state (Table 1). Also, these treatments did not have any measurable effect on the input resistance measured using small $(<0.25 \mathrm{nA})$ current pulses from the Down state (Table 1). Sharp transitions in the membrane potential between the two states were still evident after action potential currents were blocked, and the Up state maintained its square appearance and strongly preferred membrane potential near $50 \mathrm{mV}$ (data not shown).

For cells recorded with electrodes containing QX314 and D890, it was possible to test the input resistance in the $U_{p}$ and Down states without the interference of action potentials. Figure 5 shows a typical result of this test using brief depolarizing or hyperpolarizing pulses applied during the Up or Down state. As can be seen from a comparison of the top and bottom parts of Figure 5 a strong outward rectification was present at the membrane potential associated with the Up state, but was not visible unless action potentials were blocked. These observations show that QX314and D890-resistant voltage-sensitive conductances oppose the depolarization of the cell in the Up state and also oppose the hyperpolarization of the cell in the Down state. The higher input resistance of the cell in the intermediate range of membrane potentials ( -70 to $-50 \mathrm{mV}$ ), therefore, would be expected to make these potentials energetically unfavorable, and could account for the existence of preferred subthreshold membrane potentials. This behavior of the neostriatal neuron is also consistent with the results of studies of voltage-dependent potassium currents in neostriatal spiny neurons in slices (Nisenbaum et al., 1994; Nisenbaum and Wilson, 1995). These studies suggest that slowly inactivating and noninactivating voltage-sensitive potassium currents could be responsible for the high input conductance of the Up state when tested with depolarizing pulses and, thus, for the existence of the Up state as a preferred membrane potential. If this were so, poisoning potassium currents with intracellular cesium would be expected to abolish the Up state and to reveal the envelope of summating synaptic potentials filtered only by the passive properties of the spiny cell dendrite.

\section{Effect of potassium channel inhibition with intracellular cesium}

Intracellular injection of cesium had a profound effect on membrane potential transitions, primarily in the Up state. The effect of cesium depended on the concentration in the electrode. Although some broadening of action potentials was observed in electrodes filled with a combination of $\mathrm{K}$-acetate and $\mathrm{CsCl}$ or Cs-acetate, the large effects on the membrane potential fluctuations were only seen using electrodes containing cesium as the principal cation.

Cells recorded with $2 \mathrm{M} \mathrm{CsCl}$ or $2 \mathrm{M}$ Cs-acetate continued to show periods of hyperpolarization resembling the Down state, but fired action potentials at extraordinary rates and fired prolonged action potentials during the depolarizing episodes. These effects increased dramatically over the first few minutes of recording with cesium-containing electrodes and ultimately led to extreme deviations of the membrane potential, presumably because regenerative sodium and calcium currents dominated the behavior of the cell during the depolarizing episodes. During the first 10-20 min of recording, however, it was possible to record spontaneous membrane fluctuations that were not associated with such paroxysmal regenerative currents, but which showed a profound effect of intracellular cesium. The average value of the Down state was the same as controls (Table 1). This suggests that there was a relatively weak (or perhaps no) effect of intracellular cesium on the inward rectification of striatal neurons, at least during the brief recordings that could be made using cesium-filled electrodes. During the depolarizing episodes, however, the membrane potential was much more variable than that normally seen in the Up state. As a result of the noisy variability during the depolarizations, there was no preferred membrane potential, and so no clear Up state (data not shown). It was not possible to measure the Up state in cells recorded with cesium-alone electrodes, as indicated in Table 1, because there was no preferred membrane potential. Input resistance measurements were not made for the cells recorded with cesium-alone electrodes. This effect of cesium on the Up state suggested that potassium channels responsible for the outward rectification seen in the Up state after blocking action potentials may play a central role in controlling the membrane potential during synaptic activation, and thus in transforming a variable excitatory barrage into a relatively constant depolarized membrane potential. Because of the effect of cesium on releasing 
the activation of regenerative currents at the depolarized potentials, it was not possible to study spontaneous membrane potential fluctuations in cesium-treated cells for a sufficient period of time to guarantee diffusion of the ion throughout the dendrites, or to rule out a role for local regenerative sodium or calcium currents in the variability of the membrane potential during the depolarizing episodes. Thus, the membrane potential fluctuations seen during the depolarizing episodes could not be interpreted as the envelope of synaptic potentials because it might be contaminated by residual cesium-sensitive potassium currents in the dendrites or by regenerative inward currents.

For this reason, QX314 was added to all cesium-containing electrodes after collecting only a few cells and the analysis was restricted to cells recorded using cesium-filled electrodes combined with blockade of fast spikes using QX314 or blockade of all recognizable action potentials using QX314 and D890. Because there were no action potentials, it was difficult to judge the quality of recordings using cesium-filled electrodes, especially when QX314 and D890 were present. Therefore, a strict criterion for the quality of recordings was applied, requiring Down state membrane potentials of $-50 \mathrm{mV}$ and, when action potentials were not blocked (during the first few moments of the recording), at least $10 \mathrm{mV}$ of action potential overshoot. Six neurons met these criteria, half of which had action potentials blocked with a combination of QX314 (25 mM) and D890 (2 mM), and the other half contained cesium and QX314, but not D890. A much larger group of less polarized neurons was recorded, and the cesium-induced changes reported for the smaller sample here were also seen in the larger sample of neurons. For the 6 cells used, the Down state modal potential was more depolarized than for the other groups, and the Up state modal potential was much more positive (Table 1). The mean input resistance in the Down state was also higher in this group of cells than in the control or QX314/D890 groups (Table 1). The depolarization of the Down state, and the increased input resistance, was attributable primarily to a reduction in the inwardly rectifying conductance present in the Down state. It was not possible to determine whether this blockade of inward rectification was attributable to the action of cesium, or to a combination of D890 and cesium combined. In all 6 cells treated with cesium plus action potential blockers, the Up state membrane potential was greatly depolarized compared with that seen using electrodes without cesium. An example showing membrane potential fluctuations observed using QX314 and D890 to block action potentials in cesium-poisoned cells is shown in Figure 7. The membrane potential fluctuations in these cells were much more variable than those seen in control cells or those recorded with QX314- and D890-containing electrodes in the absence of cesium. Also consistent with the cesium-alone treatment was the absence of a clearly defined Up state, either in the traces or in the histogram of membrane potentials. When examined only briefly after impaling the neuron (Fig. 7, top), these cells had a more depolarized and less clearly defined Up state than seen in controls. After allowing 15-30 min for diffusion of cesium within the neurons, however, the amplitude of the depolarization gradually increased and the duration of the Down states became shorter (Fig. 8, middle). After 30-60 min of recording, the cell alternated between two extreme membrane potentials, one within a few millivolts of the Down state of control cells, and the other near $-25 \mathrm{mV}$. The membrane potential shift between Up and Down states was more than twice that seen in control cells. This temporal sequence was observed in all 6 neurons.

In these cells, it was possible to examine the effects of depolar- izing and hyperpolarizing constant currents on the spontaneous membrane potentials shifts without the interference of fast or slow action potentials. If voltage-sensitive potassium channels were responsible for converting the envelope of spontaneous synaptic excitation in spiny neurons into a relatively constant Up state, it would be expected that poisoning those channels with cesium would result in an increase in the amplitude of spontaneous depolarizations and also an increase in their variability. Although the increase in amplitude was always seen, and in fact was unexpectedly large, the increase in variability was only seen during the onset of the cesium effect. After longer exposures to cesium, a preferred membrane potential during the Up state reappeared, but at a much more depolarized level. To investigate the origin of this new Up state, the membrane potential of the neurons was manipulated using constant current. Because these cells were linearized by the treatment with QX314, D890, and cesium, it was possible to examine the spontaneous fluctuations over a wide range of imposed baseline membrane potentials. An example showing the results of this test for a cell impaled more than $40 \mathrm{~min}$ before is shown in Figure 8. As in Figures 3 and 7, $10 \mathrm{sec}$ episodes of spontaneous activity were sampled and histograms of membrane potential were constructed (data not shown). These histograms, like that at the bottom of Figure 7 , were strongly bimodal, so it was straightforward to measure the membrane potentials in the two states using the modes of the histograms. The more hyperpolarized (Down state) and depolarized (Up state) modes are shown as a function of injected current in the graph at the top right in Figure 8. Unlike the results obtained in control cells, the relationship between current and voltage was approximately linear in both states. The slope (steady-state input resistance) was much steeper during the hyperpolarized periods than during the depolarized ones, and the membrane potential fluctuations could be made to reverse polarity by passage of strong intracellular currents $(\sim 1.0 \mathrm{nA})$. The membrane potential at which reversal occurred varied between -9 and $-20 \mathrm{mV}$ for the 4 cells on which this test was performed (mean $=-15 \pm 4.7 \mathrm{mV}$ ). In the example shown in Figures 8 and 9, the reversal point was $-17 \mathrm{mV}$. The higher input resistance of the cells in the Down state was confirmed using short current pulses, as illustrated at the bottom right in Figure 8. The time constant was consistently shorter and the input resistance consistently lower in the Up state and, unlike control cells, this was independent of the polarity of the current pulse in either state. These results suggest that the depolarized state in the poisoned, linearized spiny neurons is attributable to a conductance increase with a reversal potential of -10 to $-20 \mathrm{mV}$. It should also be noted in Figure 8 that with strong hyperpolarizing constant currents, much of the variability of the depolarized state was reinstated, suggesting that the depolarized state in these poisoned cells was attributable to a saturation of that conductance as the membrane potential approached its reversal point.

The possibility that the conductance responsible for the large depolarizing episodes in the cesium-, QX314-, and D890-poisoned neurons was synaptic excitation was tested by comparing the reversal potential of the spontaneous depolarizations with that of the EPSP evoked by stimulation of the cerebral cortex. This comparison was made in all the cells with the same result. The results obtained for the same cell used in Figure 8 are illustrated in Figure 9. Three traces are shown superimposed in Figure 9, once at low sweep speed (top) and one at fast sweep speed (bottom). The lower two traces were collected in the absence of applied constant current, but one was taken while the cell was in the depolarized state and one when in the hyperpolarized state. 
2 M Cesium Acetate, $25 \mathrm{mM}$ QX-314, 2 mM D-890
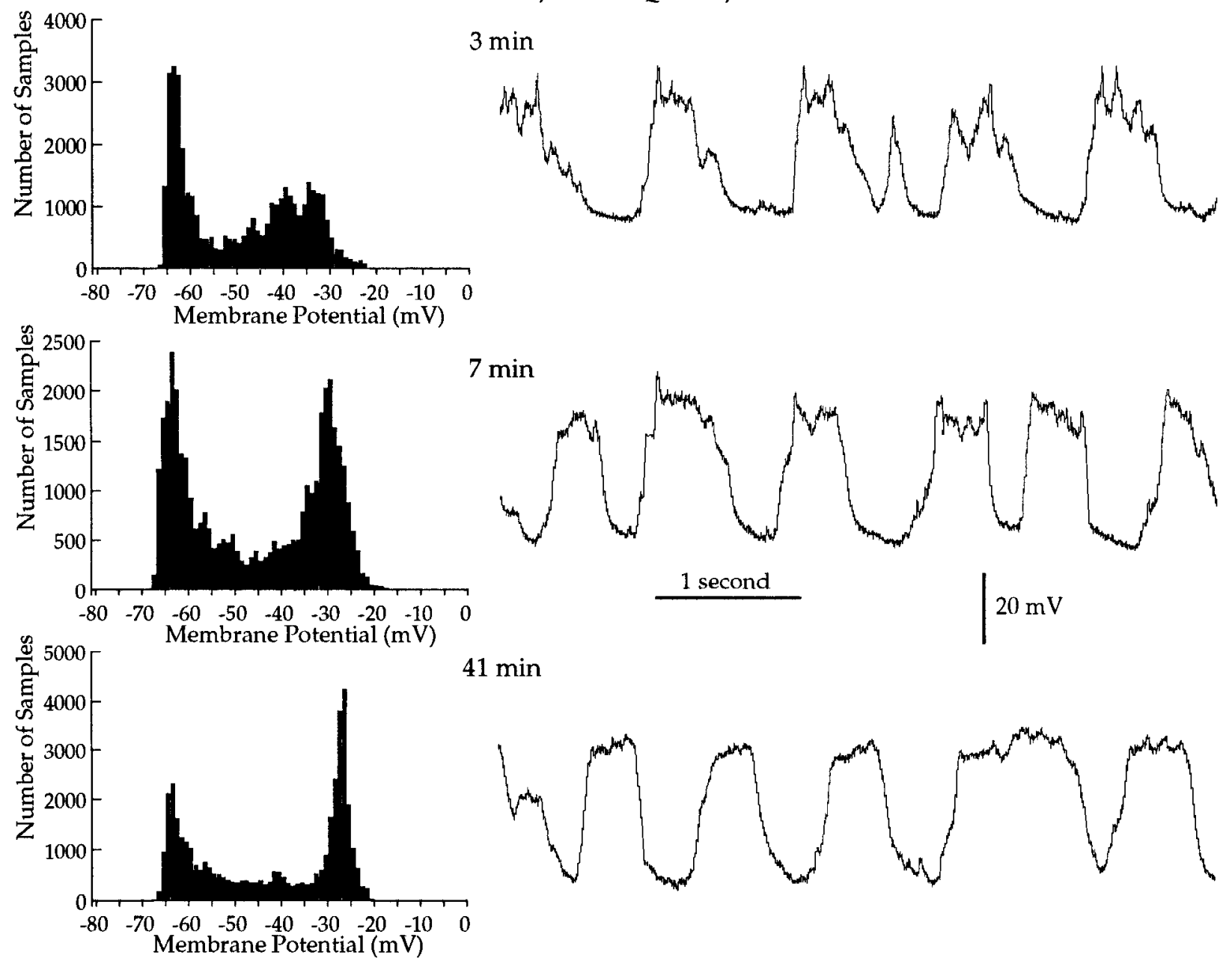

$41 \mathrm{~min}$

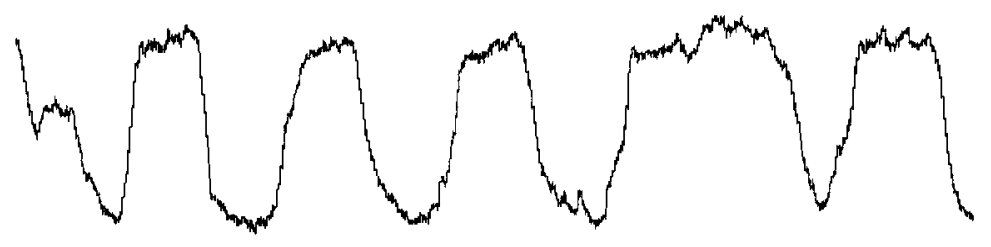

Figure 7. A change in spontaneous membrane potential fluctuations develops gradually over time after impaling neurons with electrodes filled with cesium, QX314, and D890. Initially, action potentials were blocked and membrane potential fluctuations were increased in amplitude, as in the case of QX314 and cesium, as shown in Figure 9 . Over the first $3 \mathrm{~min}$ of recording, the fluctuations increased in amplitude and began to establish a new preferred membrane potential during the depolarizing episodes (top trace and histogram). This trend continued over the next few minutes (middle trace and histogram), until the cell showed clear preferred membrane potentials during both the depolarized and hyperpolarized episodes. Instead of a preferred depolarized membrane potential around $-50 \mathrm{mV}$ seen in control cells, the preferred potential attained after 30 min or more of recording under these conditions was about $-25 \mathrm{mV}$ (bottom trace and histogram). Also, note that transitions into and out of the Down state became more gradual, so that the Down state was not associated with abrupt transitions (compare bottom with top trace).

The third trace (top-most) was taken during passage of $1.5 \mathrm{nA}$ constant depolarizing current, just positive to that required for reversal of the spontaneous membrane potential fluctuations. $\Lambda$ s previously reported for spiny cells under control conditions (Wilson, 1993), strong corticostriatal stimulation is followed by a transition to the Down state, regardless of whether the stimulus was applied during the Up or Down state. For the two traces collected in the absence of applied current, the transition to the Down state could be seen to be followed by a period of depolarization, as also expected from previous studies. The trace collected during passage of depolarizing current sufficient to reverse the spontaneous transition also reversed the slow components of the corticostriatal response. These traces, and especially the faster sweep speed versions in the lower part of Figure 9, show that during the spontaneous depolarizations, the corticostriatal EPSP is close to its reversal point, as indicated by its small amplitude. Constant current that reversed the spontaneous membrane potential shifts and the late components of the corticostriatal re- sponse also reversed the initial PSP. In the cell shown in Figures 8 and 9 , all of these reversed at the same point, $-17 \mathrm{mV}$. In three cells for which both reversal of the Up and Down states and reversal of the EPSP were examined, these occurred at the same membrane potentials $(-9,-17$, and -20 for the three different cells). Thus the large spontaneous membrane potential depolarizations seen after poisoning with cesium, QX314 and D890 are the envelope of synaptic excitation, and are so powerful that they drive the cell to within a few millivolts of the synaptic reversal potential.

\section{DISCUSSION}

\section{Membrane potential shifts underlie the firing patterns of spiny projection neurons}

Unlike giant neostriatal interneurons (Wilson et al., 1990) and many other central neurons, action potentials in neostriatal spiny projection cells are not triggered by individual synaptic potentials. They instead reflect the occurrence of membrane potential shifts 


\section{M Cesium Acetate, 25 mM QX-314, 2 mM D-890}

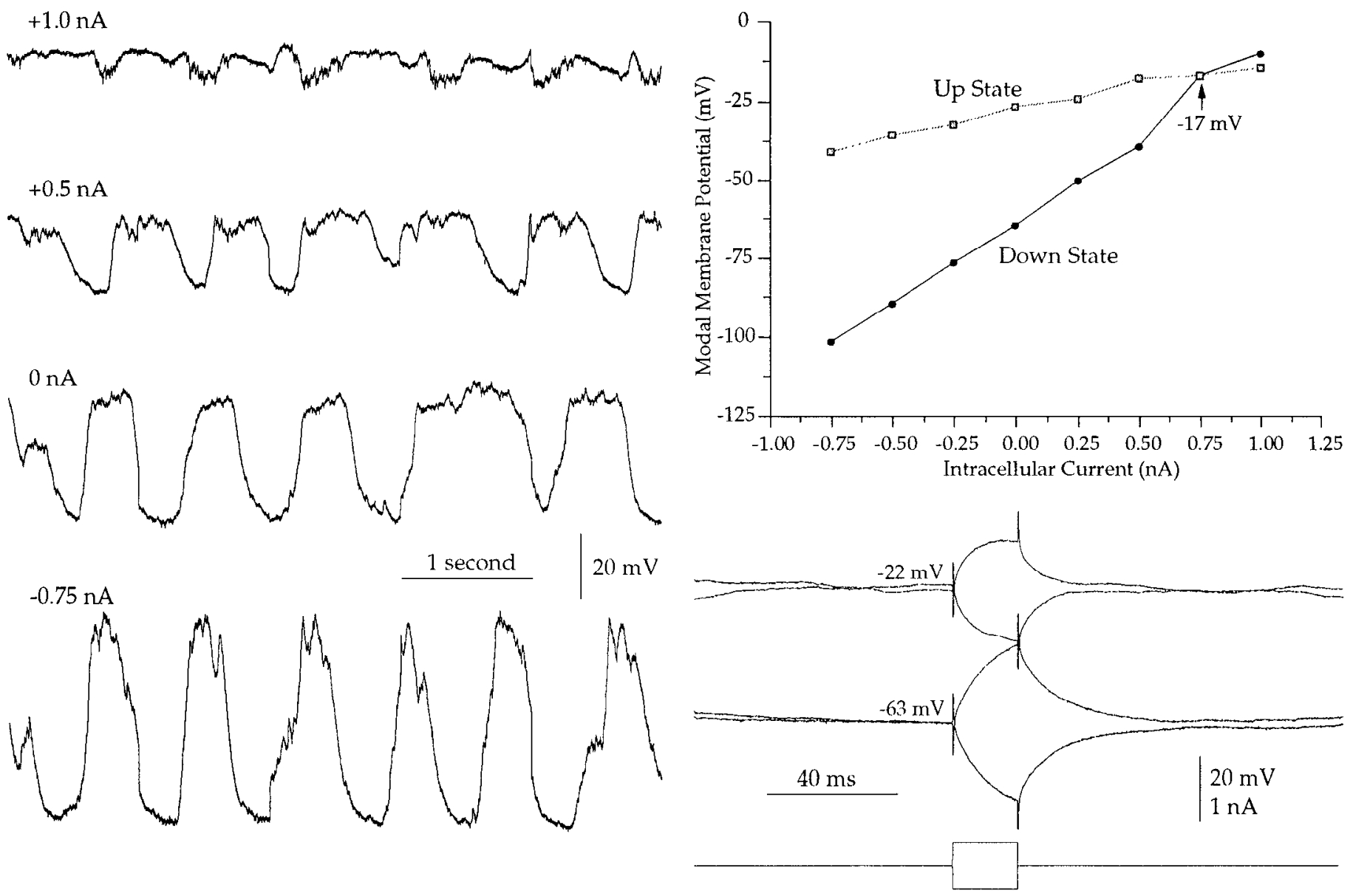

Figure 8. After linearization of the spiny neuron with cesium, D890, and QX314, the depolarizing episodes can be shown to be attributable to a conductancc incrcasc with a reversal potential of 9 to $20 \mathrm{mV}$. Traces on the left are $5 \mathrm{sec}$ of spontaneous activity, taken during passage of constant current, 40-50 min after impaling the neuron. For $10 \mathrm{sec}$ episodes, including these traces and for others at intermediate current levels, histograms like those in Figure 7 were obtained and the modal membrane potentials in the Up and Down states used to construct the current-voltage relationship shown at the top right. This is the same procedure used for control neurons to construct the graph at lower left in Figure 3. After blockade of voltagc-sensitive currents, both Up and Down state $I-V$ relationships were linear. The slope in the Up state was shallower than in the Down state, and the membrane potential fluctuations reversed at $-17 \mathrm{mV}$. The reversal is apparent in the top trace at the left, because the noisy episodes became hyperpolarizing. The whole neuron input resistance was measured by passing current pulses in the Up and Down states, and this is shown at the bottom right. The input resistance is high and the time constant long for both depolarizing and hyperpolarizing pulses applied in the Down state. The input resistance is lower and the time constant shorter in the Up state, regardless of the polarity of current. Similar tests in three other neurons under these conditions yielded reversal potentials between -9 and $-20 \mathrm{mV}$.

involving an interaction between synaptic activity in large numbers of synapses and the electrical properties of the neostriatal projection cell. Because the pattern of firing of afferents to the neostriatum arises from cortical and thalamic circuits, it is expected to be altered dramatically by anesthetics. It may therefore be argued that the pattern observed in this study may not be representative of neostriatal neurons in awake or behaving animals. Several lines of evidence suggest that although the temporal pattern of membrane potential fluctuations may be dependent upon anesthesia, the existence of two membrane potential states is a characteristic of neostriatal projection cells under a wide range of conditions. Shifts in membrane potential similar to those studied here were described briefly in early intracellular recording studies performed in decerebrate cats by Hull et al. (1970). They were also described in awake, locally anesthetized, paralyzed rats (Wilson and Groves, 1981), although the Up states were more variable in duration and less regular in occurrence than seen in anesthetized rats. In animals lightly anesthetized with barbiturates, similar shifts in membrane potential are seen, but have much shorter durations and occur periodically at $8-10 \mathrm{~Hz}$ (Calabresi et al., 1990). Although intracellular recordings from neostriatal spiny neurons in behaving animals are not available, the episodic patterns of firing of these cells in behaving monkeys closely resembles that seen in locally anesthetized rats [compare Wilson and Groves (1981) and Aldridge and Gilman (1991)]. In behaving animals, episodes of firing are even more variable in their duration and occurrence, and often are closely related to sensory stimuli, movement planning and execution, or the motivational state of the animal (Hikosaka et al., 1989; Kimura et al., 1990). These data strongly suggest that the firing in episodes by neostriatal projection neurons in behaving monkeys arises from Up state transitions similar to those seen to occur spontaneously in anesthetized rats.

\section{The origin of the Up state}

It is well established that transitions to the Up state do not occur in the absence of synaptic excitation from the cerebral cortex and 
$25 \mathrm{mM}$ QX314, 1mM D890, 2M Cs Acetate
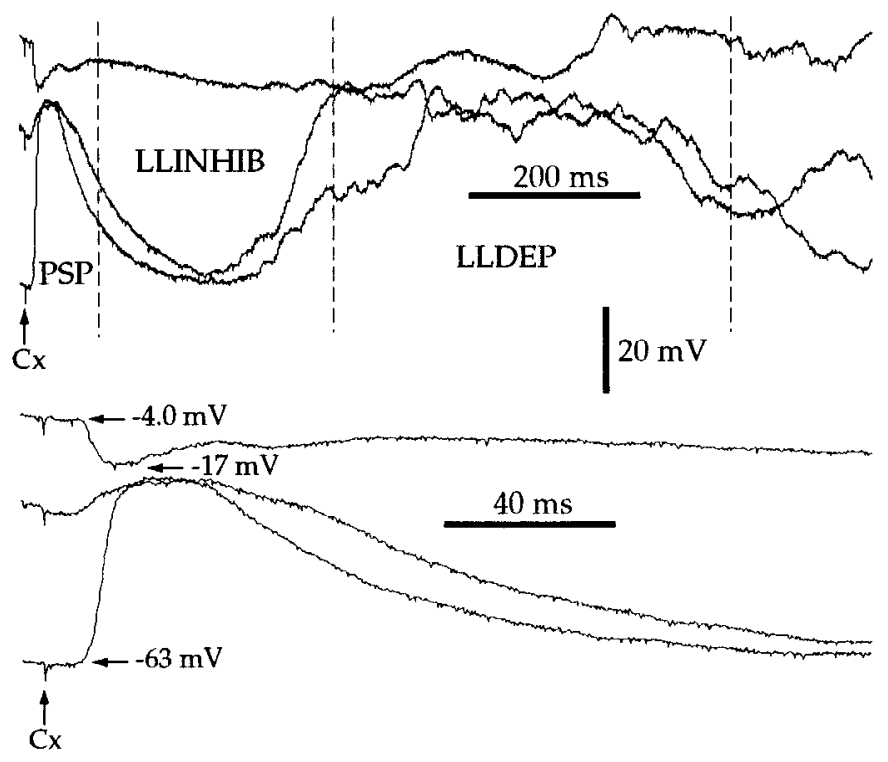

Figure 9. The reversal potential of the initial synaptic response to cortical stimulation reverses at the same potential as the Up state in cells recorded using cesium, D890, and QX314. All traces are from the same cell shown in Figure 8. The top three traces are slow sweep speed, and the bottom three traces are the same traces at faster sweep. In the top traces, the three phases of the response, the initial PSP, the long-lasting inhibition (LLINHIB), and the long-lasting depolarizing response (LLDEP) are indicated. The lower two of each group are without injected current. This pair of traces shows the response to cortical stimulation presented during the Up state and the Down state. The synaptic potential was much larger when the stimulus was presented in the Down state, suggesting that the Up state potential was near reversal for the initial PSP. Passage of $1.5 \mathrm{nA}$ depolarizing current and stimulation during the reversed Down state produced the responsc shown at the top in both the upper and lower set of traces. The initial PSP reversed near $-16 \mathrm{mV}$, and the long-lasting inhibition and subsequent depolarizing response were both reversed. This test was replicated in two other neurons using electrodes filled with cesium, D890, and QX314 and, in all cases, the reversal potentials for EPSPs and for spontaneous membrane potential shifts were identical.

thalamus. When the cortex is removed or temporarily inactivated by spreading depression, neostriatal projection neurons in vivo do not show any spontaneous activity, but remain stable in the Down state (Wilson, 1983, 1992). Iontophoretic application of cadmium, at levels that block synaptic evoked potentials in neostriatal neurons similarly abolishes spontaneous Up state transitions in vivo (Calabresi et al., 1990a). Up state transitions are not seen in ncostriatal slices, in which afferent input is interrupted (Kawaguchi et al., 1989; Calabresi et al., 1990b), and the Up state cannot be triggered in neostriatal slices either by direct depolarization or by synaptic excitation evoked by local stimulation (Wilson, 1993). There is also no biophysical mechanism available in neostriatal neurons in vivo for producing Up and Down state transitions in the absence of synaptic input. In the present study, depolarization of neustriatal cells by passing current pulses failed to reveal a second equilibrium potential at the Up state, and hyperpolarizing pulses presented in the Up state failed to reset the cell to the Down state. Poisoning inward currents carried by sodium and calcium, which offer the most likely mechanism for bistability in spiny neurons, did not alter the occurrence of spontaneous Up state transitions. These observations suggest that the Up state is not only triggered by excitatory synaptic input, but also requires sustained synaptic excitation for its maintenance. This is con- firmed by the lower input resistance seen in the Up state after poisoning the potassium conductances that normally prevent detection of the synaptic conductance changes that underlie the Up state.

Although excitation derived from cortical and thalamic inputs to the neostriatum is required for the transition to the Up state, there is no evidence that synaptic inhibition is important for maintaining the Down state. Iontophoresis of bicuculline at levels that block synaptic inhibition in vivo does not block spontaneous membrane potential shifts in vivo (Calabresi et al., 1990). This is as expected, because most or all synaptic inhibition of neostriatal projection neurons is mediated by chloride currents that reverse at levels considerably positive to the level of the Down state. In the present study, the current-voltage relationship for the Down state showed no influence of a possible synaptic chloride current but, rather, was governed primarily by the effect of an inwardly rectifying potassium conductance. A more likely time for inhibition to play a role is during the Up state, while many neostriatal neurons are likely to be active. The results of the experiments presented here do not suggest the presence of a powerful inhibition accompanying the excitation that causes the Up state. Kita et al. (1985b) showed that in slices the short-latency postsynaptic potential (PSP) response to local stimulation reverses at considerably more negative potentials than observed here, because of the presence of strong inhibitory and excitatory synaptic components of that response. The initial cortically evoked PSP observed here, and the spontaneous membrane potential shifts after linearization of the cell with cesium, reversed at about $-20 \mathrm{mV}$, indicating that these responses have a much smaller contribution from inhibitory components. Still, it is likely that both the evoked and spontaneous depolarizations have an inhibitory component, as indicated by the increase in response amplitude and Up state depolarization observed with iontophoretic administration of bicuculline (Calabresi et al., 1990a). There is no evidence for the action of synaptic inhibition during the Down state. Like corticostriatal neurons (Cowan and Wilson, 1994), neostriatal spiny neurons thus are subjected to inhibition mostly during the times of maximum excitation, rather than during their most silent periods.

Whereas the timing of transitions between the Up and Down states is determined hy excitatory synaptic input, the membrane potentials achieved during those states is not determined primarily by synaptic inputs. For the Down state this is easy to understand. The membrane potential during the Down state is determined by the inwardly rectifying potassium channel that dominates the membrane conductance of the cell in the absence of strong depolarizing synaptic currents (Kawaguchi et al., 1989; Jiang and North, 1991; Wilson, 1992; Nisenbaum and Wilson, 1995). This strong voltage-dependent conductance ensures a low input resistance in spiny neurons in the Down state, and so resists attempts by synaptic currents to alter the membrane potential. Between the Down and Up states, the inwardly rectifying conductance deactivates and a different set of voltage-sensitive channels becomes dominant. The membrane potential in the Up state is the result of an interaction between a strong depolarizing synaptic conductance and voltage-dependent currents. The results presented here indicate that of the voltage-dependent conductances, the most important are outward currents that oppose depolarization and are blocked by intracellular cesium. Although a persistent inward sodium conductance is almost certainly activated to some degree in the Up state (Kita et al., 1985a; Calabresi et al., 1987; Bargas et al., 1989; Nisenbaum et al., 1994), blockade of sodium currents with QX314 had no measurable effect on the 
average amplitude of the Up state. Studies of neostriatal slices have shown that several potassium conductances are activated in the range of membrane potentials that corresponds to the Up state (Surmeier et al., 1991; Nisenbaum and Wilson, 1995). The most recent studies indicate that a noninactivating current may be the most powerful polassium channel activated in the -55 to -45 $\mathrm{mV}$ range corresponding to the Up state (Nisenbaum et al., 1994). It is not possible to distinguish between the classes of voltageactivated potassium channels using the in vivo recording methods used here. The responses to current pulses observed in spiny neurons in vivo after cesium, QX314, and D890 poisoning resemble closely those of the same cells in slices after poisoning with extracellular TTX, cadmium, and a high ( $25 \mathrm{~mm}$ ) concentration of tetraethylammonium (Nisenbaum and Wilson, 1995), which is consistent with a block of all of the voltage-dependent potassium channels identified in those studies. But it seems unlikely that rapidly inactivating conductances could play a large role in determining the amplitude of the Up state, because this can be sustained for several seconds with a variation of only a few millivolts. The importance of voltage-dependent outward currents is indicated by the large increase in the amplitude of the Up state after such currents were poisoned by intracellular injection of cesium. After this treatment, the Up state often grew to be within a few millivolts of its reversal potential, indicating that the magnitude of synaptic conductance activated during the Up state may be many times greater than previously thought. This result suggests that the Up state is accompanied by synaptic excitation sufficient to drive the spiny cell to saturation if it were not for the action of voltagedependent potassium conductances activated by the depolarization. In recent computer simulations, it was shown that the presence of even relatively weak dendritic potassium conductances can produce the appearance of saturation of dendritic excitatory synaptic input like that observed here due to electrotonic expansion of the dendritic tree (Wilson, 1995). According to the model presented there, fluctuations of synaptic input slow enough to be tracked by potassium conductances should be smoothed out in the Up state. Increased synaptic excitation will be resisted by the increased potassium current that it evokes, while reductions in excitatory synaptic conductance that would reduce the depolarization would produce a reduction in potassium currents and a corresponding increase in the effectiveness of the remaining synaptic currents. Thus, the membrane potential achieved in the Up state, and whether it is subthreshold or suprathreshold, is as much determined by the voltage dependence of activation of potassium conductances as it is by the strength of synaptic excitation.

\section{The sharp transitions between states are attributable to nonlinear membrane properties}

The observation that the amount of time spent in the Up state can be adjusted somewhat by alteration of the membrane potential does not contradict the conclusion that the Up state is generated and maintained by excitatory synaptic input. It does indicate that the transitions between states can be affected by the membrane properties of the cells. When the cell was hyperpolarized strongly using electrodes filled with control electrolyte, no Up state transitions were seen despite the presence of episodes of synaptic excitation. This occurred because the membrane potential was forced into a range that strongly activated the inwardly rectifying potassium conductance. The input resistance of the hyperpolarized neuron was so low that even the very strong synaptic input present in these cells was unable to depolarize the cell enough to deactivate the inwardly rectifying current. Thus, the synaptic input produced an episode of noisy depolarization that was a greatly diminished image of the envelope of synaptic excitation, but never completed the transition to the Up state and never activated the currents that normally limit the amplitude of the Up state. Under more moderate levels of applied current, the amount of synaptic input required to achieve or maintain the Up state was altered by moving the membrane potential of the cell farther into the region dominated by the inward rectifier, or closer to the point of escape from its influence. Depolarizing constant current, which brought the Down state to a level at which there was little or no inward rectification, made it possible for even relatively small synaptic inputs to achieve and maintain the Up state, and thus increased the amount of time spent by the cell in that state.

Examination of the membrane potential traces taken at depolarized levels, at which the inward rectification had little influence, revealed that the transitions between the Up and Down state were less abrupt. This attests to the role of inward rectification in sharpening the transition to the Up state by resisting membrane potential shifts (Wilson, 1992). Transitions from the Up to the Down state happen in a more passive way. When excitatory synaptic input becomes too weak to maintain the cell in the Up state, decay should occur in a way resembling that seen at the offset of a current pulse. Although not completely passive because of the deactivation of outwardly rectifying conductances and the activation of inward rectification, the decay to the Down state is approximately exponential.

\section{What is signaled by the Up state transition?}

The fact that the Up and Down states reverse at the same potential as the late responses to cortical stimulation supports previous experimental results that indicated that these reflect a stimulus-induced resetting of the same mechanism that is responsible for the spontaneous membrane potential shifts. The combination of very strong synaptic input, synaptic limiting by potassium channels, and inward rectification makes the cell a subthreshold two-state device that rejects too-small inputs using the inward rectification and maintains the Up state at a relatively constant level until the input falls to a point at which the potassium channel no longer limits the response. The Up state, therefore, is an indication of sustained overwhelming afferent (mainly cortical and thalamic) barrage, and the Down state indicates periods of relative silence of inputs. The pattern of this barrage determines the durations of each state and so depends heavily on factors such as anesthesia, motor activity, and motivational state. The requirement for a relatively strong input to trigger and maintain the Up state suggests that it may require the coordinated effort of many cortical neurons. Each cortical input fiber makes few synapses with each neostriatal neuron (Wilson, 1994). Individual corticostriatal neurons show membrane potential shifts resembling those of neostriatal neurons, but generally do not fire continuously throughout their own Up states (Cowan and Wilson, 1994). Although the number of synaptic inputs required to maintain the $\mathrm{Up}$ state in a neostriatal neuron is unknown, it unquestionably requires the maintained coordinated activity of many corticostriatal cells.

\section{Conclusions}

The Up and Down states of the neostriatal neuron are a direct reflection of converging and maintained synaptic input to the cells, and the time course of the Up states reflects the envelope of the cooperative synaptic input to the cells. Because of powerful, voltage-dependent potassium currents, the membrane potential 
attained during the Up state is not a good representation of the total amount of synaptic input, but is actually determined by the voltage dependence of activation of potassium channels. Synaptic input is usually well in excess of that required to achieve the Up state. The firing of action potentials during the Up state is thus determined largely by the interplay between voltage-dependent inward and outward currents, rather than by the strength of the synaptic input. In awake animals, many neostriatal neurons undergo shifts to the Up state without firing action potentials (Wilson and Groves, 1981). When this observation was made, it was noted that this provided for two different levels of control of neostriatal projection cell firing. One was provided by the mechanisms responsible for the occurrence of the spontaneous membrane potential shifts (Up and Down membrane potential states), and one was a mechanism that determined the overall level of polarization, and thus whether or not the Up state was subthreshold or suprathreshold for action potentials. The present results indicate that the first mechanism, which has primary control of the timing of membrane potential state transitions, is directly provided by the pattern of synaptic input. The second mechanism, which determines the membrane potential in the Up state relative to the firing threshold for action potentials, is largely determined by the action of voltage-dependent intrinsic membrane properties of the spiny cell and may be subject to control by neuromodulators acting on those channels.

\section{REFERENCES}

Aldridge JW, Gilman S (1991) The temporal structure of spike trains in the primate basal ganglia: afferent regulation of bursting demonstrated with precentral cortical ablations. Brain Res 543:123-138.

Andreasen M, Hablicz JJ (1993) Local anesthetics block transient outward potassium currents in rat neocortical neurons. J Neurophysiol 69:1966-1975.

Bargas J, Galarraga E, Aceves J (1989) An early outward conductance modulates the firing latency and frequency of neostriatal neurons of the rat brain. Exp Brain Res 75:146-156.

Butterworth IV JF, Strichartz GR (1990) Molecular mechanisms of local anesthesia: a review. Anesthesiology 72:711-734.

Calabresi P, Mercuri NB, Stanzione P, Stefani A, Bernardi G (1987) Intracellular studies on the dopamine-induced firing inhibition of neostriatal neurons in vitro: evidence for $D_{1}$ receptor involvement. Neuroscience 20:757 771

Calabresi P, Mercuri NB, Stefani A, Bernardi G (1990a) Synaptic and intrinsic control of membrane excitability of neostriatal neurons. I. An in vivo analysis. J Neurophysiol 63:651-662.

Calabresi P, Mercuri NB, Bernardi G (1990b) Synaptic and intrinsic control of membrane excitability of neostriatal neurons. II. An in vitro analysis. J Neurophysiol 63:663-675.

Connors BW, Prince DA (1982) Effects of local anesthetic QX-314 on the membrane properties of hippocampal pyramidal neurons. J Pharmacol Exp Ther 220:476-481.

Cowan RH, Wilson CJ (1994) Spontaneous firing patterns and axonal projections of single corticostriatal neurons in the rat medial agranular cortex. J Neurophysiol 71:17-32.

Deisz RA, Prince DA (1987) Effect of D890 on membrane properties of neocortical neurons. Brain Res 422:63-73.

Galarraga E, Pacheco-Cano MT, Flores-Hernandez JV, Bargas J (1994) Subthreshold rectification in neostriatal spiny projection neurons. Exp Brain Res 100:239-149.

Hikosaka O, Sakamoto M, Usui S (1989) Functional properties of monkey caudate neurons. I. Activities related to saccadic eye movements. J Neurophysiol 61:780-798.
Horikawa K, Armstrong WE (1988) A versatile means of intracellular labeling: injection of biocytin and its detection with avidin conjugates. J Neurosci Methods 25:1-11.

Hull CD, Bernardi G, Buchwald NA (1970) Intracellular responses of caudate neurons to brain stem stimulation. Brain Res 22:163-179.

Jiang $Z-G$, North RA (1991) Membrane properties and synaptic responses of rat striatal neurons in vitro. J Physiol (Lond) 443:533-553.

Kawaguchi Y, Wilson CJ, Emson PC (1989) Intracellular recording of identified neostriatal patch and matrix spiny cells in a slice preparation preserving cortical inputs. J Neurophysiol 62:1052-1068.

Kimura M, Kato M, Shimazaki H (1990) Physiological properties of projection neurons in the monkey striatum to the globus pallidus. Exp Brain Res 82:672-676.

Kita T, Kita H, Kitai ST (1984) Passive electrical membrane properties of rat neostriatal neurons in an in vitro slice preparation. Brain Res 300:129-139.

Kita H, Kita T, Kitai ST (1985a) Regenerative potentials in rat neostriatal neurons in an in vitro slice preparations. Exp Brain Res 60:54-62.

Kita T, Kita H, Kitai ST (1985b) Local stimulation induced GABAergic response in rat striatal slice preparations: intracellular recording on QX-314 injected neurons. Brain Res 360:304-310.

Kita T, Kita H, Kitai ST (1985c) Effects of 4-aminopyridine (4-AP) on rat neostriatal neurons in an in vitro slice preparation. Brain Res 361:10-18.

Nisenbaum ES, Wilson CJ (1995) Potassium currents responsible for inward and outward rectification in rat neostriatal spiny projection neurons. J Neurosci 15:4449-4463.

Nisenbaum ES, Wilson CJ, Surmeier DJ (1994a) Depolarization evokes a slowly-activationg $\mathrm{K}^{+}$current in rat striatal neurons. Soc Neurosci Abstr 20:564.

Nisenbaum ES, Xu ZC, Wilson CJ (1994b) Contribution of a slowlyinactivating potassium current to the transition to firing of neostriatal spiny projection neurons. J Neurophysiol 71:1174-1189.

Pineda JC, Galarraga E, Bargas J, Cristancho M, Aceves J (1992) Carybdotoxin and ampamin sensitivity of the calcium-dependent repolarization and afterhyperpolarization in neostriatal neurons. J Neurophysiol 68:287-294

Surmeier DJ, Foehring R, Stefani A, Kitai ST (1991) Developmental expression of a slowly-inactivating voltage dependent potassium current in rat neostriatal neurons. Neurosci Lett 122:41-46.

Surmeier DJ, Xu ZC, Wilson CJ, Stefani A, Kitai ST (1992) Grafted neostriatal neurons express a late-developing transient potassium current. Neuroscience 48:849-856.

Uchimura N, Cherubini E, North RA (1989) Inward rectification in rat nucleus accumbens neurons. J Neurophysiol 62:1280-1286.

Valvidia HH, Coronado R (1990) Internal and external effects of dihydropyridines in the calcium chammel of skeletal muscle. J Gen Physiol 95:1-27.

Wilson CJ (1992) Dendritic morphology, inward rectification and the functional properties of neostriatal neurons. In: Single neuron computation (McKenna T, Davis J, Zornetzer SF, eds). pp 141-171. San Diego: Academic.

Wilson CJ (1993) The generation of natural firing patterns in neostriatal neurons. In: Chemical signaling in the basal ganglia. Prog Brain Res 99:277-297.

Wilson CJ (1994) The contribution of cortical neurons to the firing pattern of striatal spiny neurons. In: Models of information processing in the basal ganglia (Houk JC, Davis JL, Beiser DG, eds), pp 29-50. Cambridge: MIT.

Wilson CJ (1995) Dynamic modification of dendritic cable properties and synaptic transmission by voltage-gated potassium channels. J Comput Neurosci 2:91-115.

Wilson CJ, Groves PM (1981) Spontancous firing patterns of identificd spiny neurons in the rat neostriatum. Brain Res 220:67-80.

Wilson CJ, Chang HT, Kitai ST (1983) Disfacilitation and long-lasting inhibition of neostriatal neurons in the rat. Exp Brain Res 51:227-235. 